\title{
Assessment of Dentists' Knowledge Concerning the Management of Breastfeeding Patients in Dental Office
}

\author{
Aranka Ilea ${ }^{1}$, Adela Cristina Lazări ${ }^{1}$, Adriana Emanuela Morar ${ }^{2}$, Adina Bianca Boșca*3, Dan Buhățel ${ }^{1}$, \\ Anca Ionel ${ }^{1}$, Claudia Nicoleta Feurdean ${ }^{1}$, Anida Maria Băbțan ${ }^{1}$, Nausica Bianca Petrescu', Ioana \\ Codruța Mirică ${ }^{1}$, Willi Andrei Uriciuc ${ }^{1}$, Roxana Ioana Bordea ${ }^{1}$, Arin Sava ${ }^{1}$, Radu Septimiu Câmpian ${ }^{1}$ \\ and Alina Simona Sovrea ${ }^{3}$
}

${ }^{1}$ Department of Oral Rehabilitation, Oral Health and Dental Office Management, "Iuliu Hațieganu" University of Medicine and Pharmacy Cluj-Napoca, Romania

${ }^{2}$ Postgraduate student of Faculty of Dentistry, "Iuliu Hațieganu” University of Medicine and Pharmacy Cluj-Napoca, Romania ${ }^{3}$ Department of Histology, Faculty of Medicine, "Iuliu Hațieganu" University of Medicine and Pharmacy Cluj-Napoca, Romania

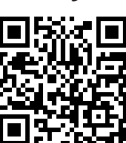

*Corresponding author: Bianca Adina Boșca, Department of Histology, Faculty of Medicine, "Iuliu Hațieganu" University of Medicine and Pharmacy Cluj-Napoca, Romania

ARTICLE INFO

Received: 坢 March 01, 2019

Published: March 08, 2019

Citation: Aranka Ilea, Adela Cristina Lazăr, Adriana Emanuela Morar, Adina Bianca Boșca, Dan Buhățel, Anca Ionel, et al. Assessment of Dentists' Knowledge Concerning the Management of Breastfeeding Patients in Dental Office. Biomed J Sci \& Tech Res 15(4)-2019. BJSTR. MS.ID.002736.

Keywords: Breastfeeding; Nursing; Infant; Dental Treatment; Dentistry; Dental Procedures

\section{ABSTRACT}

Introduction: Breastfeeding is the feeding of babies and young children with milk from a woman's breast. Breastfeeding has numerous health benefits, including the lower risk for respiratory tract infections, asthma, diarrhea, food allergies, type 1 diabetes, and leukemia. It may also improve cognitive development and prevent obesity in adulthood.

Purpose: To assess the dentists' medical knowledge regarding the dental treatment allowed during the breastfeeding period.

Methodology: In this study were included active dentists. An online questionnaire was applied. The questionnaire was anonymous and the responses were collected online. The questions assessed the therapeutic approach of the patients during the breastfeeding period, what kind of treatments they perform in this category of patients and the time chosen for the dental treatments. The last category of questions collected data about the medication used or prescribed in breastfeeding patients. The data was analyzed using the descriptive statistics and the results were presented as means.

Results: More than half of the doctors surveyed would treat a breastfeeding woman in any emergency situations, only if she postpone breastfeeding for the next 24 hours. About $85 \%$ of the respondents will perform the anesthesia in a nursing woman, but about just $1 / 3$ will use mepivacaine and less than $3 \%$ will use lidocaine, anesthetic substances admitted in a breastfeeding woman. In our study, about $90 \%$ of doctors who indicated the administration of an antibiotic to a breastfeeding woman chose a synthetic penicillins drug that are admitted during the lactation period.

Conclusion: Most dentists have insufficient knowledge regarding the treatments that can be performed in breastfeeding women. Therefore, post-graduate courses are required to update the knowledge of dentists in this domain.

\section{Introduction}

Breastfeeding, also known as nursing, is the feeding of babies and young children with milk from a woman's breast [1]. Health professionals recommend that breastfeeding begin within the first hour of a baby's life and continue as often and as much as the baby wants [2,3]. During the first few weeks of life babies can be nursed roughly every two to three hours and the duration of a feeding is usually ten to fifteen minutes on each breast [4]. Older children can be breastfed less often [5]. Mothers may express their milk using breast pumps, so that it can be used later 
when breastfeeding is not possible [1]. Breastfeeding has a number of benefits to both mother and baby, that infant formula lacks $[3,6]$. Deaths of an estimated 820,000 children under the age of five could be prevented globally every year by increasing breastfeeding [7]. Breastfeeding decreases the risk of respiratory tract infections and diarrhoea, both in developing and developed countries [2,3]. Other benefits include lower risks of asthma, food allergies, type 1 diabetes, and leukemia [3]. Breastfeeding may also improve cognitive development and prevent obesity in adulthood [2]. Mothers may feel pressured to breastfeed, but in the developed world, children generally can grow up normally when are bottle fed [8].

Benefits for the mother include: less blood loss following delivery, better uterus shrinkage, and decreased postpartum depression [3]. Breastfeeding delays the return of menstruation and fertility, a phenomenon known as lactational amenorrhea [3]. Long term benefits for the mother include decreased risk of breast cancer, cardiovascular disease, and rheumatoid arthritis $[3,7]$. Moreover, breastfeeding is less expensive than infant formula $[9,10]$. Health organizations, including the World Health Organization (WHO), recommend breastfeeding exclusively in the first six months $[2,3,11]$. This means that no other foods or drinks other than possibly vitamin D are typically given [11]. After the introduction of solid foods at six months of age, recommendations include continued breastfeeding until one to two years of age or more [2,3]. Globally about 38\% of infants are only breastfed during their first six months of life [2]. In the United States in 2015, 83\% of women begin breastfeeding and 58\% were still breastfeeding at 6 months, although only 25\% exclusively [12]. Medical conditions that contraindicate breastfeeding are rare [3]. Mothers who take certain recreational drugs and medications should not breastfeed (e.g. - antiretroviral medications, chemotherapy agents, sleep-aid medicine, lithium and lamotrigine) [13]. Smoking, limited amounts of alcohol, or coffee are not reasons to avoid breastfeeding [14-16].

\section{Aim}

To assess the dentists' knowledge regarding the dental treatments that can be performed in breastfeeding women and how to prescribe drugs for these patients in the dental office.

\section{Materials and Methods}

An online questionnaire regarding the management of breastfeeding patients in the dental office was administered through the www.isondaje.ro platform such as was described by Ilea et. al [17]. The study was attended by 128 dentists, of various specialties, aged between 25 and 55 years. The questionnaire was anonymous and the responses were collected online. The first category of questions assessed the therapeutic approach of the breastfeeding patients, what kind of treatments they perform in this category of patients and the time chosen for the dental treatments. The questions about the dental treatments, endodontic treatment for pulp pathology, specific treatment for gingivitis and chronic periodontitis, and the dental extractions performed in a breastfeeding patient had five variants of response:

a) In emergency cases only

b) After the breastfeeding period ceased

c) I would perform such treatment at any time, but without anesthesia

d) I would perform the treatments at any time, and if anesthesia is necessary, I recommend the expression and the discarding of the next milk

e) I perform dental treatments at any time and if anesthesia is required, I choose an anesthetic that is compatible with breastfeeding

The second category of questions evaluated the doctors' recommendation to perform dental $\mathrm{x}$-rays in breastfeeding patients. The last category of questions evaluated the medication used or prescribed for breastfeeding patients. When asked about the use of anesthetic substances in a breastfeeding woman, there were only two variants of response: I do not perform anesthesia in a breastfeeding woman, or yes, I do the anesthesia, followed by an open response to be completed the most commonly used anesthetic. Data were analyzed using Microsoft Excel and the results were exemplified using descriptive statistics.

\section{Results}

\section{The Attitude of Dentists Regarding Treatments that Can Be Performed in a Breastfeeding Patient}

The question referring to the attitude adopted at the time of the anamnesis when they are informed that the patient in the dental chair is breastfeeding, had four variants of response:

a) I do not perform any dental treatment;

b) If it is an emergency, I send her to the specialized hospital service;

c) I have a reserved attitude and treat only those conditions that do not require anesthesia;

d) I treat a breastfeeding woman like any other patient. Of the total of 128 subjects, no dentist chose not to treat a nursing patient

e) one dentist (1\%) chose the second option

f) 55 doctors (43\%) chose the third option

g) and 72 subjects (56\%) chose to treat a pregnant woman like any other patient

h) It can be seen that the last answer was chosen by more than half of the subjects (Figure 1). 


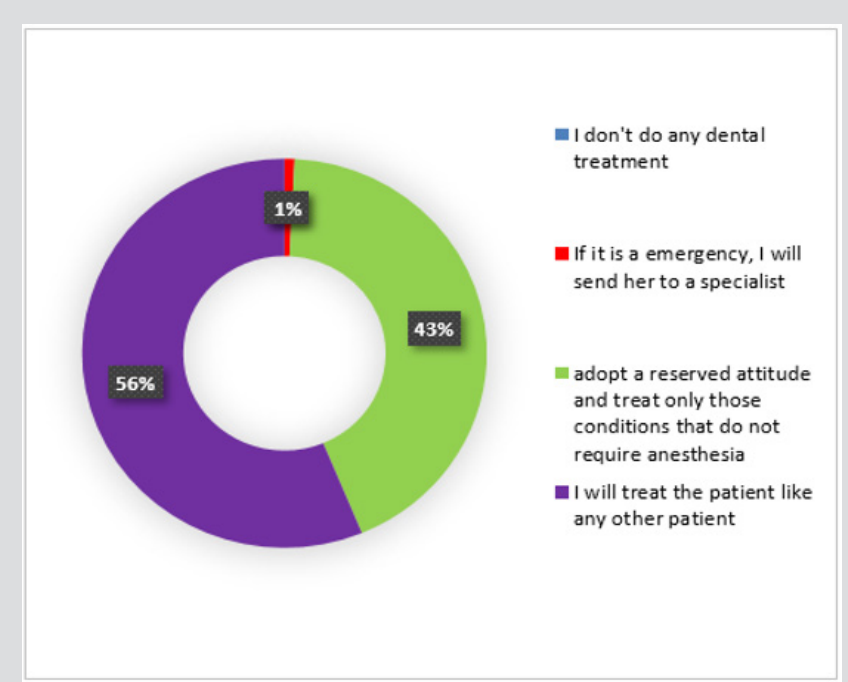

Figure 1: The graphical representation of dentists' attitude towards a breastfeeding patient.

\section{The Reasons Why a Dentist Would Not Treat a Patient Who is Breastfeeding}

The next question in the questionnaire was addressed only to subjects who chose not to treat a breast-fed patient or had reserved attitude, which was one of the first three variants of the previous question:

a) I do not perform any dental treatment,

b) If it is an emergency, I send her to the hospital specialty service or

c) I have a reserved attitude and treat only those conditions that do not require anesthesia.

There were four possible answers to the question "What are the reasons why you do not treat a nursing patient?":

a) I do not want to be responsible if some complications arise;

b) I take into account the benefit-risk ratio and if the risks are greater than the benefits, I do not perform any treatment;

c) fear; and

d) other reasons (open-ended variation). Only seven responses were collected. Two doctors (29\%) chose the first response

e) four doctors (57\%) chose the second variant

f) none of the doctors chose the fear, as a reason of not treating a breastfeeding patient, and one doctor (14\%) selected the last option

g) and responded that the reason for not treating a nursing patient was that the anesthetic is excreted in breast milk (Figure 2).

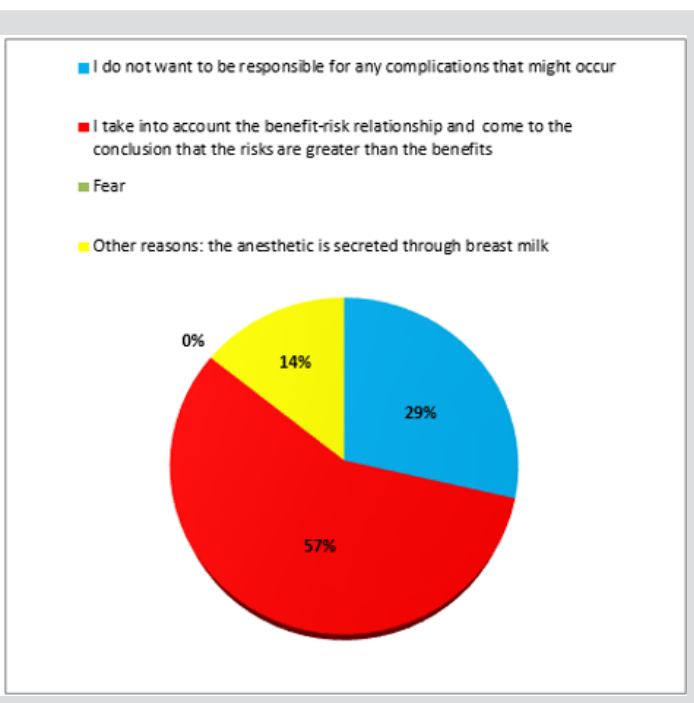

Figure 2: The graphical representation of the dentist's reasons for not treating a breastfeeding patient.

\section{Dental Treatments in a Breastfeeding Patient}

When asking about the dental treatments performed in a breastfeeding patient, there were 128 responses, out of which 5 responses (4\%) were the first variant

a) There was no response for the second option

b) 11 responses $(8.7 \%)$ were the third option

c) 79 responses $(62.7 \%)$ were the fourth option

d) and 31 responses (24.6\%) were the last option

e) Therefore, most dentists would perform dental treatments at any time, but would recommend to the patient not to breastfeed after the anesthesia and to discard the next milk. About a quarter of the respondents would choose an anesthetic compatible with breastfeeding (Figure 3).

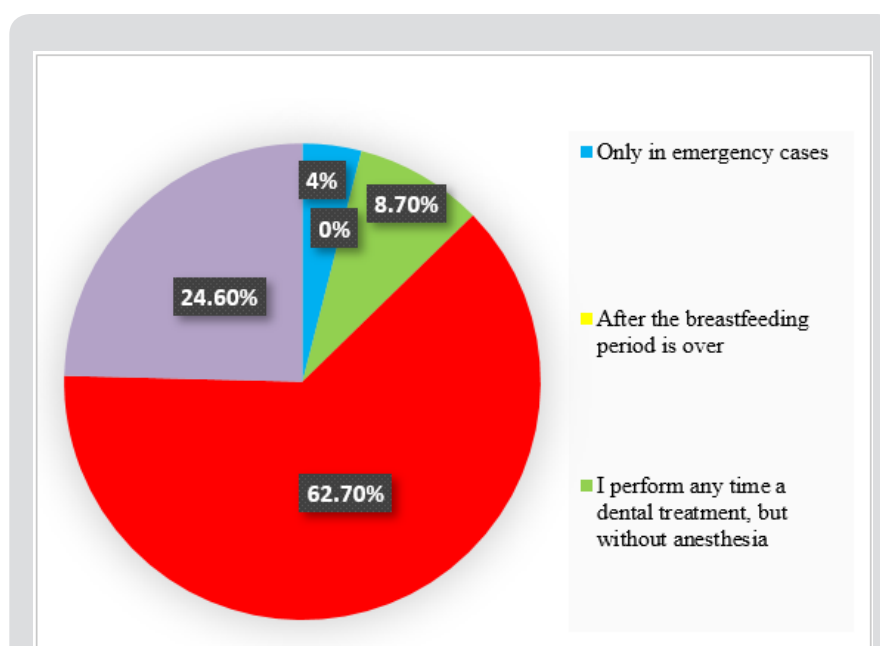

Figure 2: The graphical representation of dental treatments performed in a breastfeeding woman. 


\section{Endodontic Treatments in a Nursing Patient}

When asking about the endodontic treatment (for pulpitis or pulp necrosis) in a breastfeeding patient, there were 128 responses, out of which 6 responses (4.8\%) were the first variant

a) 1 response $(0.8 \%)$ was the second variant

b) 7 responses $(5.6 \%)$ were the third option

c) 82 responses (65\%) were the fourth variant

d) and 30 responses (23.8\%) were the last variant

e) Most doctors would perform an endodontic treatment at any time but would recommend to the patient that after the anesthesia they should express milk and discard it. Almost a quarter of the respondents would choose an anesthetic that is compatible with breastfeeding.

\section{Specific Treatment for Gingivitis in a Breastfeeding Patient}

The question about the specific treatment for gingivitis in a breastfeeding patient had a total of 128 responses, out of which six answers $(4.7 \%)$ were the first variant

a) two answers (1.6\%) were the second option

b) 20 answers $(15.9 \%)$ were the third variant

c) 64 answers $(50.8 \%)$ were the fourth variant

d) and 34 answers (27\%) were the last variant

e) Half of physicians would perform the specific treatment for gingivitis at any time but would recommend the discard of the milk after the anaesthesia. More than a quarter of the respondents would choose an anaesthetic that is compatible with breastfeeding.

\section{Specific Treatment for Chronic Periodontitis in a Nursing Patient}

The question about the periodontal treatment in a breastfeeding patient, had a total of 128 responses, of which 6 responses $(4.8 \%)$ were the first variant

a) one response $(0.8 \%)$ was the second variant

b) 11 responses (8.6\%) were the third variant

c) 75 responses $(58.6 \%)$ were the fourth variant

d) and 35 responses (27.2\%) were the last variant. More than half physicians would perform periodontal treatment at any time, but would recommend the expression and discarding of the next milk. More than a quarter of the respondents would choose an anesthetic that is compatible with breastfeeding.

\section{Dental Extractions in Breastfeeding Patients}

When asking about the dental extraction performed in a breastfeeding patient, there were 128 answers, out of which 17 answers (13.3\%) were the first variant

a) 5 responses (3.9\%) were the second variant

b) none of the doctors chose the variant

c) 77 responses $(55.5 \%)$ were the fourth variant

d) and 35 responses (27.3\%) were the last variant

e) More than half physicians would perform an extraction at any time but would recommend that the patient should express and discard the next milk. More than a quarter of the respondents would choose an anesthetic that is compatible with breastfeeding (Figure 4).

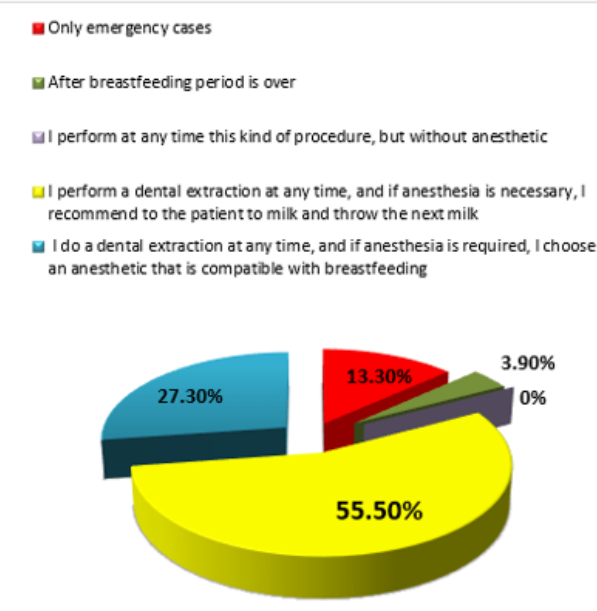

Figure 4: The graphical representation of dental extractions performed in a breastfeeding patient.

\section{Performing Dental X-Rays in a Breastfeeding Patient}

When asked about recommending dental x-rays in a breastfeeding patient, there were four variants of answer:

a) No;

b) Yes, if she passed the breastfeeding period;

c) Yes, but then I recommend not to breastfeed for a while and then express and discard the next milk;

d) Yes, anytime, without special recommendations. Of the total of 125 responses, 11 responses (8.8\%) were the first variant

e) 9 responses (7.2\%) were the second variant

f) 13 responses $(10.4 \%)$ were the third variant

g) and 92 responses (73.6\%) were the last variant (Figure 5). The answer to this question showed that regarding to dental radiographs in a nursing patient, about $3 / 4$ of the doctors were well informed. 


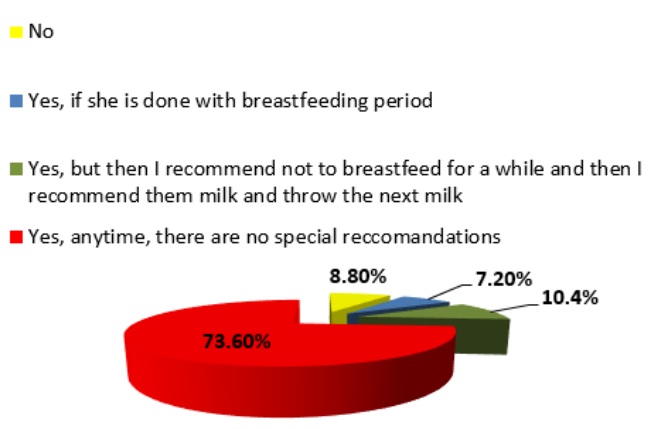

Figure 5: The graphical representation of $\mathrm{x}$-rays performed in a breastfeeding patient.

\section{Use of Anesthetics in a Breastfeeding Patient}

The question about the use of anesthetic substances in a breastfeeding woman had two variants of response:

a) I do not perform anesthesia in a breastfeeding woman; and

b) Yes, I perform the anesthesia, which was followed by an open question regarding the most commonly used anesthetic. Of the 128 responses, 19 responses (14.8\%) were the first variant

c) 109 responses $(85.2 \%)$ were the second variant

d) In the second variant, to the open question regarding the anesthetic substances used, the 109 responses were divided as follows: articaine $4 \%$ solution (68.8\%), mepivacaine $(27.5 \%)$, lidocaine $(2.75 \%)$ and any anesthetics (1.83\%) (Figure 6). Most of the respondents will perform the anesthesia in a nursing woman, but about just $1 / 3$ will use mepivacaine and less than $3 \%$ will use lidocaine, anesthetic substances admitted in a breastfeeding woman.

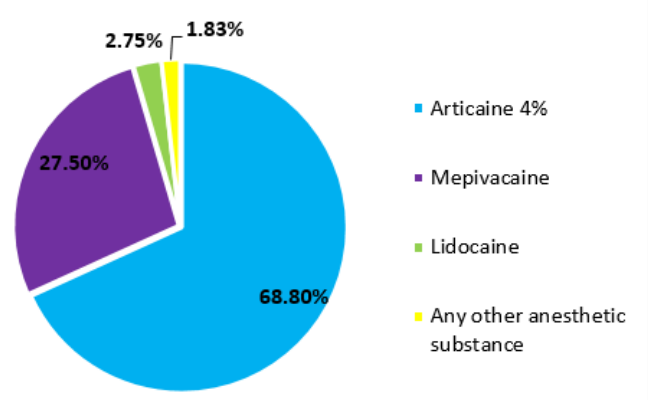

Figure 6: The graphical representation of the most frequently used anesthetic substances in a breastfeeding woman

\section{Prescription of Antibiotics in a Breastfeeding Patient}

The question about prescribing antibiotics to a breastfeeding patient had two variants of response:

\section{a) No and}

b) Yes, which was followed by an open question regarding the most frequently prescribed antibiotic. Out of the 128 answers, 74 answers (57.8\%) were the first variant

c) and 54 answers (42.2\%) were the second variant

d) Regarding the most frequently prescribed antibiotics, the 54 responses were divided as follows: amoxicillinclavulanic acid (48\%), amoxicillin (31\%), ampicillin (7\%), cefuroxime (3.6\%), other cephalosporins (1.8\%), clindamycin (1.8\%), prescribed only after consulting the pediatrician or neonatologist (5\%), prescribing only after consulting the gynecologist (1.8\%). Almost half of the doctors would prescribe the same type of antibiotic which they usually prescribe in the dental office. About $90 \%$ of doctors who indicated the administration of an antibiotic to a breastfeeding woman chose a synthetic penicillins drug that are admitted during the lactation period.

\section{Discussions}

The hormonal changes associated with breastfeeding were discussed with reference to the findings from animal studies. Both lactation and stimulation of breastfeeding are phenomena involving hormones in the anterior and posterior lobe of the pituitary gland. In women and animals, complex hormonal changes occur during pregnancy, being represented by the estrogen and progesterone, reaching peak levels towards the end of pregnancy. The most important postpartum hormone is prolactin, which initiates lactation. And the decrease in estrogen and progesterone levels, once the fetus and the placenta are delivered, helps to stimulate lactation [18]. An indirect effect of the high levels of prolactin, which supports breastfeeding, is the increased need for calcium in the nursing woman. Some authors stated that during breastfeeding, 300-400 mg of calcium are transferred to breast milk, while other authors have stated that during lactation, calcium is preserved by the kidney to maintain the bone metabolism. Bone calcium loss is counterbalanced by higher levels of vitamin $\mathrm{D}$, growth hormone changes, prolactin, and also by nutritional habits and lifestyle $[19,20]$. After birth, the patient is in a recovery period, called period of lice. This physiological period lasts about six to eight weeks and is necessary for the body to be restored after birth.

In our study, referring to the dental treatments, most dentists would perform it at any time, but would recommend to the patient not to breastfeed and express and discard the next milk after the anesthesia. The main and most important issue raised in the dental office is the administration of drugs. In our study, almost half of the doctors would prescribe the same type of medicine which they usually prescribe in the dental office, probably based on their knowledge regarding the properties of the drug. The prescription of medication during breastfeeding is a challenge for both the patient and the physician. Therefore, many mothers are advised to express and discard the breast milk if they take certain medication. 
However, this advice is often based on limited medical information. Some studies reviewed the evidence on certain medicines that are safe or others that should be avoided during breastfeeding. Dentists should be familiarized with the risks and benefits of prescribing for a nursing patient certain classes of drugs: antibiotics, local anesthetics, and emergency medications [21].

The milk secretion occurs 24-48 hours after birth. Most substances or drugs ingested by the mother are secreted into the breast milk. Antibiotics can be ingested by the baby through breast milk if the mother is under antibiotic treatment for a variable period of $5-7$ - 10 days required as adjuvant treatment of oral cavity pathology. This results in early exposure of the infant to antibiotics, which will reduce the long-term breastfeeding beneficial effects (such as reducing the frequency of infections and the risk of overweight) and also, the infant's microbiota can be modified [22]. In our study, about $90 \%$ of doctors who indicated the administration of an antibiotic to a nursing woman chose a synthetic penicillins drug that are admitted during the lactation period. Almost half of them indicated the administration of amoxicillin-clavulanic acid. Although the concentration of this antibiotic is low in breast milk in order to produce infant toxicity $(0.68-1.3 \mu \mathrm{g} / \mathrm{ml}$ when administering $1 \mathrm{~g}$ of amoxicillin - clavulanic acid), there is the possibility of hypersensitization of the infant to this drug and the alteration of the intestinal microbiota, so this should be avoided [23].

Some authors recommend that after dental treatments that require the loco-regional anesthesia, the baby's first suckling should be suppressed, and milk be expressed and discarded [24]. In our study, most dentists would perform a dental treatment at any time but would recommend to the patient not to breastfeed after the anesthesia. It is important to know how the drugs are secreted into the breast milk and what factors are involved, in order to know what drugs can be safely administered. According to the literature, the use of lidocaine, mepivacaine, and bupivacaine for local anesthesia is considered compatible with breastfeeding because of low levels in breast milk. Thus, the relative infant dose (RID), which is calculated by dividing the infant's dose via milk by the mother's dose, is $0.5-3.1$ for lidocaine and 0.9 for bupivacaine. In our study, approximately two-thirds of physicians indicated local anesthesia with articaine and only about $1 / 3$ with mepivacaine, which showed insufficient knowledge among respondents [25]. Multiple situations requiring pharmacological treatment may occur in breastfeeding women. Due to the many health benefits of breast milk in infants, breastfeeding should only be discontinued when the drug necessary for the mother could be harmful to the child. There is no reliable evidence that most drugs cause adverse effects to the breastfed child. Moreover, interruption of breastfeeding, even temporary, may be difficult. Therefore, decisions on the use of medication should be based on correct information. Most antibiotics are considered compatible with breastfeeding [26].

\section{Conclusion}

There is insufficient knowledge of an appreciable number of dentists in related to the treatments that can be performed in breastfeeding women. Therefore, postgraduate courses and continuous medical education would be required in order to update the knowledge of dental practitioners.

\section{Acknowledgment}

Aranka Ilea, Adela Cristina Lazăr and Adriana Emanuela Morar have equals contributions as first authors of the article.

\section{References}

1. Andreas NJ, Kampmann B, Mehring Le Doare (2015) Human breast milk: A review on its composition and bioactivity. Early Hum Dev 91: 629-635.

2. Martin CR, Ling PR, Blackburn GL (2016) Review of Infant Feeding: Key Features of Breast Milk and Infant Formula. Nutrients 8(5): 279.

3. Bagga N, Nadipineni R, Mohamed A, Poddutoor P, Chirla DK (2018) A quality initiative to improve exclusive breast milk feeding in preterm neonates. Int J Pediatr Adolesc Med 5(4): 131-134.

4. Ingram J, Johnson D, Copeland M, Churchill C, Taylor H (2015) The development of a new breastfeeding assessment tool and the relationship with breastfeeding self-efficacy. Midwifery 31(1): 132-137.

5. Ip S, Chung M, Raman G, Trikalinos TA, Lau J (2009) A summary of the agency for healthcare research and quality's evidence report on breastfeeding in developed countries. Breastfeeding Med 4(1): 17-30.

6. Victora CG, Bahl R, Barros AJ, França GV, Horton S, et al. (2016) Lancet Breastfeeding Series Group. Breastfeeding in the 21st century: epidemiology, mechanisms, and lifelong effect. Lancet 387(10017): 475490.

7. Lawrence RA, Lawrence RM (2011) Breastfeeding: A Guide for the Medical Profession. Elsevier Health Sciences. 2011, The ( $7^{\text {th }}$ edn). Mosby, an imprint of Elsevier Inc, pp. 227-228.

8. Ballard O, Morrow AL (2013) Human Milk Composition: Nutrients and Bioactive Factors. Pediatr Clin North Am 60(1): 49-74.

9. (1997) Breastfeeding and the use of human milk. Pediatrics 100(6): 1035-1039.

10. Bzikowska Jura A, Czerwonogrodzka Senczyna A, Oledzka G, Szostak Wegierek D, Weker $\mathrm{H}$, et al. (2018) Maternal Nutrition and Body Composition During Breastfeeding: Association with Human Milk Composition. Nutrients 10(1379): 1-15.

11. Kramer MS, Kakuma R (2012) Optimal duration of exclusive breastfeeding. The Cochrane Database of Systematic Reviews 8(8): CD003517.

12. (2018) Results: Breastfeeding Rates. Center for Disease Control and Prevention (CDC)

13. (2013) Are there any special conditions or situations in which I should not breastfeed? National Institutes of Health.

14. May PA, Hasken JM, Blankenship J, Marais AS, Joubert B, et al. (2016) Breastfeeding and maternal alcohol abuse: prevalence and effects on child outcomes and fetal alcohol spectrum disorders. Reprod Toxicol 63: 13-21.

15. Wright MJ, Bentley ME, Mendez MA, Adair LS (2015) The interactive association of dietary diversity scores and breast-feeding status with weight and length in Filipino infants aged 6-24 months. Public Health Nutr 18(10): 1762-1773.

16. Chamberlain C, OMara Eves A, Oliver S, Caird JR, Perlen SM, et al. (2013) Psychosocial interventions for supporting women to stop smoking in pregnancy. Cochrane Database Syst Rev 23(10): CD001055. 
17. Ilea A, Lazăr AC, Morar AE, Boșca AB, Băbțan AM, et al. (2019) Assessment of Dentists' Knowledge Concerning the Management of Pregnant Women in the Dental Office. Biomed J Sci \& Tech Res 15(2): 1-9.

18. Voogt J (1978) Control of hormone release during lactation. Clin Obstet Gynaecol 5(2): 435-455.

19. Kovacs CS (2001) Calcium and bone metabolism in pregnancy and lactation. J Clin Endocrinol Metab 86(6): 2344-2348.

20. Kalkwarf HJ (1999) Hormonal and dietary regulation of changes in bone density during lactation and after weaning in women. J Mammary Gland Biol Neoplasia 4(3): 319-329.

21. Hotham N, Hotham E (2015) Drugs in breastfeeding. Australian Prescriber 38(5): 156-159.

22. Korpela K, Salonen A, Virta LJ, Kekkonen RA, De Vos WM (2016) Association of Early-Life Antibiotic Use and Protective Effects of

\section{ISSN: 2574-1241}

DOI: 10.26717/BJSTR.2019.15.002736

Bianca Adina Boșca. Biomed J Sci \& Tech Res

This work is licensed under Creative Commons Attribution 4.0 License

Submission Link: https://biomedres.us/submit-manuscript.php
Breastfeeding: Role of the Intestinal Microbiota. JAMA Pediatr 170(8): 750-757.

23. De Sá Del Fiol F, Barberato Filho S, de Cássia Bergamaschi C, Lopes LC, Gauthier TP (2016) Antibiotics and Breastfeeding. Chemotherapy 61(3): 134-143.

24. Donaldson M, Goodchild J (2012) Pregnancy, breast-feeding and drugs used in dentistry. Am J Dent Assoc 143(8): 858-871.

25. Smathers AB, Collins S, Hewer I (2016) Perianesthetic Considerations for the Breastfeeding Mother. J Perianesth Nurs 31(4): 317-329.

26. Bar Oz B, Bulkowstein M, Benyamini L, Greenberg R, Soriano I, et al. (2003) Use of antibiotic and analgesic drugs during lactation. Drug Safety 26(13): 925-935.

BIOMEDICAL
RESEARCHES $\quad \begin{aligned} & \text { Assets of Publishing with us } \\ & \text { - Global archiving of articles } \\ & \text { IsSN: } 2574-1241\end{aligned}$

